\title{
Is the lateral jack-knife position responsible for cases of transient neurapraxia?
}

\author{
Diana Margarita Molinares, MD,' Timothy T. Davis, MD, ${ }^{1}$ Daniel A. Fung, MD, ${ }^{1}$ \\ John Chung-Liang Liu, MD, ${ }^{2}$ Stephen Clark, PT, DPT, ${ }^{3}$ David Daily, $\mathrm{PT},{ }^{3}$ and James M. Mok, MD ${ }^{4}$ \\ ${ }^{1}$ Orthopedic Pain Specialists, Santa Monica; ${ }^{2}$ Departments of Neurosurgery and Orthopedic Surgery and Spine Center, Keck \\ Medicine of USC, Los Angeles; ${ }^{3}$ Athletic Physical Therapy, Westlake Village; and ${ }^{4}$ The Spine Institute, Santa Monica, California
}

\begin{abstract}
OBJECTIVE The lateral jack-knife position is often used during transpsoas surgery to improve access to the spine. Postoperative neurological signs and symptoms are very common after such procedures, and the mechanism is not adequately understood. The objective of this study is to assess if the lateral jack-knife position alone can cause neurapraxia. This study compares neurological status at baseline and after positioning in the $25^{\circ}$ right lateral jack-knife (RLJK) and the right lateral decubitus (RLD) position.

METHODS Fifty healthy volunteers, ages 21 to 35 , were randomly assigned to one of 2 groups: Group A (RLD) and Group B (RLJK). Motor and sensory testing was performed prior to positioning. Subjects were placed in the RLD or RLJK position, according to group assignment, for 60 minutes. Motor testing was performed immediately after this 60 -minute period and again 60 minutes thereafter. Sensory testing was performed immediately after the 60-minute period and every 15 minutes thereafter, for a total of 5 times. Motor testing was performed by a physical therapist who was blinded to group assignment. A follow-up call was made 7 days after the positioning sessions.
\end{abstract}

RESULTS Motor deficits were observed in the nondependent lower limb in $100 \%$ of the subjects in Group B, and no motor deficits were seen in Group A. Statistically significant differences $(p<0.05)$ were found between the 2 groups with respect to the performance on the 10-repetition maximum test immediately immediately and 60 minutes after positioning. Subjects in Group B had a 10\%-70\% (average 34.8\%) decrease in knee extension strength and 20\%-80\% (average $43 \%$ ) decrease in hip flexion strength in the nondependent limb.

Sensory abnormalities were observed in the nondependent lower limb in $98 \%$ of the subjects in Group B. Thirty-six percent of the Group B subjects still exhibited sensory deficits after the 60 -minute recovery period. No symptoms were reported by any subject during the follow-up calls 7 days after positioning.

CONCLUSIONS Twenty-five degrees of right lateral jack-knife positioning for 60 minutes results in neurapraxia of the nondependent lower extremity. Our results support the hypothesis that jack-knife positioning alone can cause postoperative neurological symptoms.

http://thejns.org/doi/abs/10.3171/2015.3.SPINE14928

KEY WORDS neurapraxia; jack-knife; positioning complications; technique

$\mathrm{I}$ $\mathrm{N}$ the past few years, minimally invasive surgical (MIS) techniques have been described as a less morbid alternative to the traditional open approaches for lumbar interbody fusions. Less tissue trauma, shorter operative and recovery time, and less operative pain are some advantages of the MIS approaches. ${ }^{19,21}$ Lateral transpsoas interbody fusion is a MIS approach that was first described in 2001 as an alternative to the anterior approach. ${ }^{1,19,21}$ This approach has decreased risk of vascular injury and retrograde ejaculation as compared with the anterior lumbar interbody fusion approach. ${ }^{1,22}$ The small incision required in this tech- nique also leads to less postoperative pain and a shorter recovery time..$^{19,21,22}$

The transpsoas technique is used for the treatment of a wide variety spine conditions, including degenerative spinal disorders, scoliosis, trauma, infections, and tumors. ${ }^{1,28}$ In the past few years, multiple articles have documented the outcomes of this technique. Rodgers et al. studied 600 patients with degenerative spine conditions who underwent lateral interbody fusion. With at least 1 year of follow-up, patients in this study reported $86.7 \%$ satisfaction. ${ }^{22}$ Similar results were observed in a smaller study,

ABBREVIATIONS MIS = minimally invasive surgical; RLD = right lateral decubitus; RLJK = right lateral jack-knife; tEMG = triggered electromyography; 10 Max test = 10-repetition maximum test.

SUBMITTED September 19, 2014. ACCEPTED March 25, 2015.

INCLUDE WHEN CITING Published online September 11, 2015; DOI: 10.3171/2015.3.SPINE14928. 
in which Ozgur et al. reported a 91\% fusion rate and $75 \%$ clinical satisfaction..$^{20}$ However, complications have also been associated with this procedure. Psoas weakness, quadriceps weakness, and thigh paresthesias have been reported postoperatively. ${ }^{1,19,22,27}$ Lumbar plexus injury and psoas trauma have been considered as possible causes of these symptoms. ${ }^{1,19,27}$ The transpsoas approach avoids manipulation of major vessels but causes manipulation of the lumbar plexus during dilation and retraction., ${ }^{1,16,21,33}$ Many anatomical studies have reported the most narrow access point and the highest density of neural structures to be at the L4-5 level. 5,13,16,31 Based on a cadaveric study, one publication recommended special attention to the retractor placement and dilation time due to the proximity and displacement of neural structures. ${ }^{5}$ Neuromonitoring systems can identify motor structures with triggered electromyography (tEMG) probes during disc localization but cannot identify sensory branches or give good feedback regarding neural integrity as the case progresses.

Despite tEMG, postoperative neurological "complications" have been reported at rates ranging from $8 \%$ to $30 \%$. Most of these symptoms have been reported to resolve within 6 weeks to 6 months. ${ }^{25}$ The variability in spite of a consistent technique and instrumentation system suggests that there may be other causes affecting the neural structures. Several articles in the literature propose operative positioning as a source of postoperative neurological symptoms..$^{9,15,25,35-37}$ Stretch and/or compression of the neural structures and the consequent decreased perfusion of the nerve can contribute to postoperative deficits. ${ }^{6,9,17,25,31}$ The lateral transpsoas approach requires the patient to be in the lateral decubitus position with the table jack-knifed $25^{\circ}$ to $30^{\circ} .{ }^{21,28}$ The objective of this study is to investigate whether the lateral jack-knife position alone can cause neurological symptoms similar to those reported in the literature after transpsoas lumbar fusion.

\section{Methods}

Approval for this study was obtained from Quorum Review.

After review board approval was obtained, 55 healthy volunteers were screened and 50 (ages 21-35 years) were enrolled. Exclusion criteria included pregnancy, previous back surgery, vasculitis, neuropathies, back conditions (scoliosis, spondylolisthesis, vertebral disc herniation, spinal stenosis, congenital deformities, back pain), > 10-year history of diabetes mellitus, joint disease, heavy smoking or alcohol consumption, and body mass index (BMI) > 40. The 5 subjects excluded were excluded due to scoliosis (2), recent knee surgery, recent tear of the anterior cruciate ligament, and chronic back pain.

Two visits were scheduled for each subject, at least 24 hours apart. During the first visit, subjects were screened and provided informed consent. A baseline strength test was performed on each subject's bilateral lower extremities for hip flexion (psoas) and knee extension (quadriceps). Strength testing was performed by a certified physical therapist who was blinded to the subject's participation status. A 10-repetition maximum test (10Max test) was selected, as it is an established, objective, and reproducible way to measure subjects' strength. ${ }^{10,34}$ The maximum weight each subject could lift during knee extension and hip flexion 10 times with full range of motion was determined in 3 or fewer tries to avoid muscle fatigue. A 3-minute break was given between each attempt. Once the maximum baseline weight was determined, a second visit was scheduled, with at least a 24-hour interval to prevent cumulative muscle fatigue. During the second visit, baseline sensory testing, which included light touch and pinprick, was performed to determine any area of numbness and/or paresthesias in the dermatomes of the lower extremities. The baseline sensory testing was performed by a nonblinded physician (D.M.) who was overseeing and conducting the study. This physician was not blinded to group assignment because we attempted to be consistent and have all sensory testing performed by a single examiner. We were unable to blind this tester because the onset of sensory deficits was recorded while the patients were in their assigned positions. The results of the baseline sensory testing were considered normal in all subjects, and there were no exclusions during this phase. Subjects were then randomly assigned to one of the two study groups using GraphPad Software. Group A (right lateral decubitus [RLD], Fig. 1) and Group B (right lateral jack-knife [RLJK], Fig. 2) and placed in the assigned position.

Subjects in Group B (RLJK) were initially positioned in the right lateral decubitus position (left side up), then the table was bent $25^{\circ}$ to increase the space between the ribs and the hips on the nondependent (left) side, in addition to $40^{\circ}$ to $45^{\circ}$ hip and knee flexion. Subjects in Group A (RLD) were kept in the right lateral decubitus position. Subjects in both groups remained in the positions for 60 minutes; customary positioning technique was applied using tape and pressure point relief. During this time, subjects' symptoms were documented. A sensory examination (postpositioning sensory test) was performed immediately after the 60-minute positioning session and every 15 minutes thereafter, for a total of 5 times. The 10Max test was performed using the individual subject's baseline weight limit-one attempt immediately after positioning and another 60 minutes after the positioning session (a 3-minute break between knee extension and hip flexion was used to avoid muscle fatigue). All strength testing was performed and documented by the same physical therapist, who was blinded to which group the subject was assigned to. Strength testing was performed in the gym with the necessary strength testing equipment,

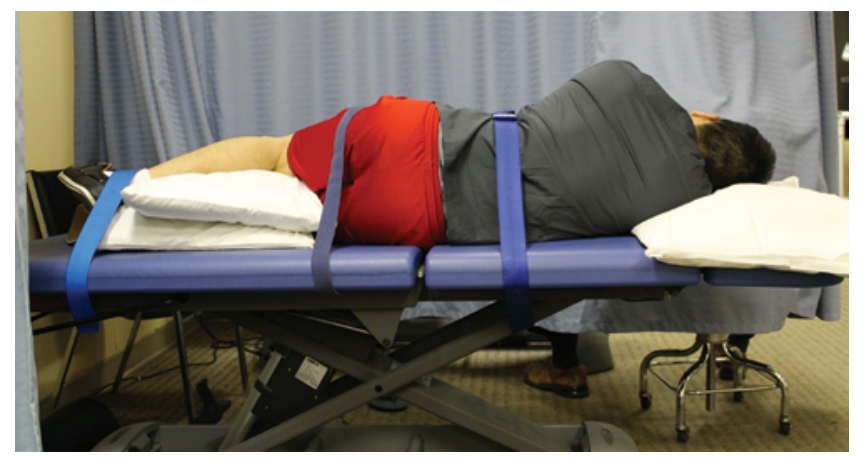

FIG. 1. Right lateral decubitus position. Figure is available in color online only. 


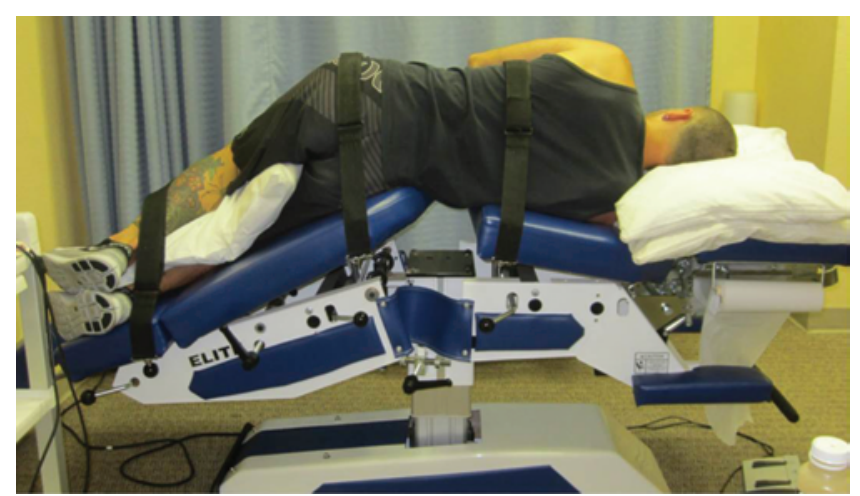

FIG. 2. Right lateral jack-knife position. Figure is available in color online only.

whereas the positioning was performed in a closed treatment room. Seven days after the second visit, subjects received a phone call to follow up on their symptoms.

\section{Statistics}

Statistical analysis was performed using SAS statistical software (SAS Institute Inc.). Fisher analysis was used to determine the difference in sensory examination findings between the two groups. The results obtained every 15 minutes were systematically compared. The Student t-test was used to determine the between-groups differences in the results of the 10Max test, immediately and 60 minutes after the positioning time.

\section{Results}

Fifty volunteers participated in this study-24 women and 26 men between 21 and 35 years old (average age 28.2 years) (Table 1). The average weight, height, and BMI were very similar between the 2 groups; the overall BMI average was 23.59. None of the participants had a BMI greater than $40 \mathrm{~kg} / \mathrm{m}^{2}$ (Table 1). During the postpositioning time, $100 \%$ of the subjects in Group B (RLJK) had decreased strength in the nondependent (left) lower extremity, without any significant differences in results between males and females. Immediately after the positioning session, all the subjects in Group B (RLJK) had 10\%-70\% (average of $34.8 \%$ ) decrease in 10Max nondependent knee extension, while the subjects in the Group A (RLD) had no deficit $(p=0.0012)$. Similar results were observed in Group B nondependent hip flexion, with a 20\%-80\% (average of $43 \%$ ) decrease in 10Max, while no deficits were observed in Group A (RLD) ( $<$ 0.001). Group B also demonstrated some motor impairment in the dependent lower extremity. Eleven subjects in Group B (RLJK) had a 10\%-40\% (average $15 \%$ ) decrease in 10Max on the dependent knee extension and 5 subjects had a 10\% decrease in 10Max in dependent hip flexion, while no motor changes were detected in the dependent lower extremity for Group A (RLD) subjects. However, no statistically significant differences were observed on the dependent side $(\mathrm{p}=0.15)$ (Table 2).

Sixty minutes after the positioning time, the 10Max test was performed again. Although there was improvement, Group B subjects continued to exhibit weakness in the nondependent lower extremity. Sixty-four percent of
TABLE 1. Demographic characteristics of the study participants

\begin{tabular}{ccc}
\hline Characteristic & Group A (RLD) & Group B (RLJK) \\
\hline Sex & & 12 \\
\hline Female & 11 & 13 \\
\hline Male & 14 & \\
\hline Age $(\mathrm{yrs})$ & & 28.1 \\
\hline Mean & 28.4 & $21-35$ \\
\hline Range & $25-34$ & 66.1 \\
\hline Weight $(\mathrm{kg})$ & & \\
\hline Mean & 70.96 & 1.67 \\
\hline Range & $46.72-102.05$ & $1.44-1.8$ \\
\hline Height $(\mathrm{m})$ & & \\
\hline Mean & 1.73 & 23.57 \\
\hline Range & $1.54-1.91$ & $16.82-39.38$ \\
\hline BMl $\left(\mathrm{kg} / \mathrm{m}^{2}\right)$ & &
\end{tabular}

$\mathrm{BMI}=$ body mass index.

Group B subjects continued to demonstrate a 10\%-60\% (average 20\%) decrease in 10Max on the nondependent knee extension (10\% improvement compared with the initial postpositioning 10Max test) and 68\% exhibited $10 \%-60 \%$ (average $25 \%$ ) decrease in the 10Max hip flexion (12\% improvement compared with the first postpositioning test). There was no dependent limb deficit in knee extension ( $p=0.0028)$ or hip flexion $(p<0.001)$ in Group A. None of the participants in Group A or Group B had deficits in the dependent lower extremity (Table 3).

Sensory testing was performed immediately after the positioning session and every 15 minutes thereafter for a 60 -minute period (5 times total). The responses were classified in 3 categories: 1) absent, when no sensation was reported by the subject; 2) decreased, the sensation was present but not normal; and 3) normal, when the subject reported full sensation. Sensory function was tested based on dermatomal distributions. Most subjects in Group B had sensory changes in the nondependent lower limb during the immediate postpositioning testing. L-1, L-2, and L-3 were the most affected dermatomes on pinprick (Table 4). Ninety-two percent of the participants had changes (absent or decreased response) during the initial postpositioning pinprick examination in the L-1 dermatome, $88 \%$ in $\mathrm{L}-2$, and $44 \%$ in L-3. After the 60-minute recovery time, $36 \%$ of Group B still had changes in pinprick response in the L- 1 and L-2 dermatomes. These results were found to be significantly different from those obtained in Group A, where no alteration in sensory function (no change in sensory testing response) was observed in the nondependent lower limb ( $p=0.00001)$. In the dependent lower limb, response to pinprick remained normal in both groups.

Similar results were observed for the light touch sensory test. Decreased light touch sensation was observed immediately after positioning in $80 \%$ of Group B subjects in the nondependent L1 dermatome, while $68 \%$ had abnormal sensations in the L-2 dermatome, $52 \%$ in L-3, and $8 \%$ at L-4. None of the subjects in Group B had any 
TABLE 2. Results of motor testing performed immediately after 60-minute positioning session*

\begin{tabular}{|c|c|c|c|c|c|c|c|}
\hline \multirow{2}{*}{$\begin{array}{c}\text { Subject } \\
\text { No. }\end{array}$} & \multirow{2}{*}{$\begin{array}{l}\text { Age } \\
\text { (yrs) }\end{array}$} & \multirow[b]{2}{*}{ Sex } & \multirow[b]{2}{*}{ Position } & \multicolumn{4}{|c|}{ PostP 10Max } \\
\hline & & & & RKE & LKE & RHF & LHF \\
\hline 1 & 29 & $\mathrm{~F}$ & RLD & $10 / 10$ & $10 / 10$ & $10 / 10$ & $10 / 10$ \\
\hline 2 & 33 & $M$ & RLJK & $10 / 10$ & $8 / 10$ & $10 / 10$ & $8 / 10$ \\
\hline 3 & 27 & $M$ & RLJK & $10 / 10$ & $3 / 10$ & $10 / 10$ & $5 / 10$ \\
\hline 4 & 27 & $\mathrm{~F}$ & RLJK & $10 / 10$ & $7 / 10$ & $10 / 10$ & $5 / 10$ \\
\hline 5 & 28 & $\mathrm{~F}$ & RLD & $10 / 10$ & $10 / 10$ & $10 / 10$ & $10 / 10$ \\
\hline 6 & 28 & M & RLJK & $10 / 10$ & $6 / 10$ & $10 / 10$ & $6 / 10$ \\
\hline 7 & 26 & M & RLJK & $10 / 10$ & $9 / 10$ & $9 / 10$ & $2 / 10$ \\
\hline 8 & 31 & $\mathrm{M}$ & RLD & $10 / 10$ & $10 / 10$ & $10 / 10$ & $10 / 10$ \\
\hline 9 & 30 & $\mathrm{~F}$ & RLD & $10 / 10$ & $10 / 10$ & $10 / 10$ & $10 / 10$ \\
\hline 10 & 25 & M & RLD & $10 / 10$ & $10 / 10$ & $10 / 10$ & $10 / 10$ \\
\hline 11 & 29 & $\mathrm{~F}$ & RLJK & $9 / 10$ & $5 / 10$ & $9 / 10$ & $7 / 10$ \\
\hline 12 & 27 & $M$ & RLD & $10 / 10$ & $10 / 10$ & $10 / 10$ & $10 / 10$ \\
\hline 13 & 30 & $\mathrm{~F}$ & RLJK & $9 / 10$ & $8 / 10$ & $10 / 10$ & $5 / 10$ \\
\hline 14 & 27 & M & RLJK & $10 / 10$ & $9 / 10$ & $10 / 10$ & $7 / 10$ \\
\hline 15 & 30 & $\mathrm{M}$ & RLD & $10 / 10$ & $10 / 10$ & $10 / 10$ & $10 / 10$ \\
\hline 16 & 26 & $M$ & RLD & $10 / 10$ & $10 / 10$ & $10 / 10$ & $10 / 10$ \\
\hline 17 & 27 & $M$ & RLD & $10 / 10$ & $10 / 10$ & $10 / 10$ & $10 / 10$ \\
\hline 18 & 28 & $\mathrm{~F}$ & RLJK & $10 / 10$ & $9 / 10$ & $10 / 10$ & $7 / 10$ \\
\hline 19 & 32 & $\mathrm{~F}$ & RLJK & $10 / 10$ & $6 / 10$ & $10 / 10$ & $5 / 10$ \\
\hline 20 & 34 & $\mathrm{~F}$ & RLD & $10 / 10$ & $10 / 10$ & $10 / 10$ & $10 / 10$ \\
\hline 21 & 21 & $\mathrm{~F}$ & RLJK & $9 / 10$ & $8 / 10$ & $10 / 10$ & $10 / 10$ \\
\hline 22 & 25 & $M$ & RLD & $10 / 10$ & $10 / 10$ & $10 / 10$ & $10 / 10$ \\
\hline 23 & 23 & $\mathrm{~F}$ & RLJK & $10 / 10$ & $7 / 10$ & $10 / 10$ & $9 / 10$ \\
\hline 24 & 30 & $F$ & RLD & $10 / 10$ & $10 / 10$ & $10 / 10$ & $10 / 10$ \\
\hline 25 & 25 & $\mathrm{~F}$ & RLD & $10 / 10$ & $10 / 10$ & $10 / 10$ & $10 / 10$ \\
\hline 26 & 29 & $M$ & RLJK & $10 / 10$ & $8 / 10$ & $10 / 10$ & $9 / 10$ \\
\hline 27 & 27 & $\mathrm{~F}$ & RLJK & $10 / 10$ & $8 / 10$ & $10 / 10$ & $7 / 10$ \\
\hline 28 & 31 & $\mathrm{~F}$ & RLD & $10 / 10$ & $10 / 10$ & $10 / 10$ & $10 / 10$ \\
\hline 29 & 31 & $M$ & RLJK & $9 / 10$ & $6 / 10$ & $10 / 10$ & $7 / 10$ \\
\hline 30 & 21 & $\mathrm{~F}$ & RLJK & $8 / 10$ & $5 / 10$ & $9 / 10$ & $2 / 10$ \\
\hline 31 & 25 & $\mathrm{~F}$ & RLJK & $8 / 10$ & $4 / 10$ & $10 / 10$ & $4 / 10$ \\
\hline 32 & 29 & $\mathrm{~F}$ & RLJK & $9 / 10$ & $6 / 10$ & $10 / 10$ & $8 / 10$ \\
\hline 33 & 27 & $\mathrm{~F}$ & RLJK & $10 / 10$ & $5 / 10$ & $9 / 10$ & $2 / 10$ \\
\hline 34 & 29 & $M$ & RLD & $10 / 10$ & $10 / 10$ & $10 / 10$ & $10 / 10$ \\
\hline 35 & 27 & $\mathrm{~F}$ & RLD & $10 / 10$ & $10 / 10$ & $10 / 10$ & $10 / 10$ \\
\hline 36 & 28 & $\mathrm{~F}$ & RLD & $10 / 10$ & $10 / 10$ & $10 / 10$ & $10 / 10$ \\
\hline 37 & 32 & $M$ & RLD & $10 / 10$ & $10 / 10$ & $10 / 10$ & $10 / 10$ \\
\hline 38 & 29 & $M$ & RLJK & $10 / 10$ & $8 / 10$ & $10 / 10$ & $8 / 10$ \\
\hline 39 & 28 & $M$ & RLD & $10 / 10$ & $10 / 10$ & $10 / 10$ & $10 / 10$ \\
\hline 40 & 28 & $M$ & RLD & $10 / 10$ & $10 / 10$ & $10 / 10$ & $10 / 10$ \\
\hline 41 & 27 & $\mathrm{~F}$ & RLD & $10 / 10$ & $10 / 10$ & $10 / 10$ & $10 / 10$ \\
\hline 42 & 29 & $M$ & RLJK & $9 / 10$ & $6 / 10$ & $10 / 10$ & $3 / 10$ \\
\hline 43 & 30 & $M$ & RLJK & $9 / 10$ & $6 / 10$ & $10 / 10$ & $6 / 10$ \\
\hline 44 & 31 & $M$ & RLD & $10 / 10$ & $10 / 10$ & $10 / 10$ & $10 / 10$ \\
\hline 45 & 27 & $M$ & RLD & $10 / 10$ & $10 / 10$ & $10 / 10$ & $10 / 10$ \\
\hline 46 & 26 & $M$ & RLD & $10 / 10$ & $10 / 10$ & $10 / 10$ & $10 / 10$ \\
\hline 47 & 29 & $\mathrm{~F}$ & RLD & $10 / 10$ & $10 / 10$ & $10 / 10$ & $10 / 10$ \\
\hline
\end{tabular}

TABLE 2. Results of motor testing performed immediately after 60-minute positioning session* (continued)

\begin{tabular}{|c|c|c|c|c|c|c|c|}
\hline \multirow{2}{*}{$\begin{array}{c}\text { Subject } \\
\text { No. }\end{array}$} & \multirow{2}{*}{$\begin{array}{l}\text { Age } \\
\text { (yrs) }\end{array}$} & \multirow[b]{2}{*}{ Sex } & \multirow[b]{2}{*}{ Position } & \multicolumn{4}{|c|}{ PostP 10Max } \\
\hline & & & & RKE & LKE & RHF & LHF \\
\hline 48 & 29 & $\mathrm{M}$ & RLJK & $6 / 10$ & $7 / 10$ & $10 / 10$ & $8 / 10$ \\
\hline 49 & 35 & M & RLJK & $10 / 10$ & $9 / 10$ & $10 / 10$ & $9 / 10$ \\
\hline 50 & 31 & $\mathrm{~F}$ & RLJK & $8 / 10$ & $6 / 10$ & $9 / 10$ & $5 / 10$ \\
\hline
\end{tabular}

$\mathrm{LHF}=$ left hip flexion; $\mathrm{LKE}=$ left knee extension; PostP = postpositioning; RHF $=$ right hip flexion; $\mathrm{RKE}=$ right knee extension.

* Shading indicates Group B (RLJK).

change in response to light touch in the dependent lower limb. Response to light touch was normal in the bilateral lower limbs in all Group A subjects $(p=0.0007)$. After the 60 minutes of recovery, a significant improvement in the response to light touch was observed in Group B. Just 36\% of the subjects in this group had an abnormal response to light touch in the L-1 dermatome, $12 \%$ in the $\mathrm{L}-2$, and $8 \%$ in the L-3 dermatome (Table 5). The response to light tough remained normal in all subjects in Group A.

At the 7-day posttesting follow-up phone call, all subjects had returned to their baseline asymptomatic state.

\section{Discussion}

Since the 1930s, when the first description of lumbar interbody fusion was published by Capener and colleagues, a significant number of techniques have been described for various spinal conditions. ${ }^{28}$ Open anterior interbody fusion was used for several years with good results, but the high number of vascular complications during this approach encouraged Cloward et al. to publish the technique of posterior lumbar interbody fusion..$^{28,32}$ This technique, however, was later associated with nerve root injury and irritation, and transforaminal lumbar interbody fusion was described as a modification. ${ }^{25,28}$ MIS approaches for spine surgery have been expanded in an attempt to decrease disruption to surrounding structures. Shorter surgical time, lower blood loss, decreased postoperative pain, and shorter recovery are some of the advantages attributed to the minimally invasive spine surgery.1,14,22,22,32 Endoscopic dissection seemed to open a new chapter in spine surgery when it was described in 1991, but complex technique caused for a steep learning curve. ${ }^{19,21}$ A decade later, a new approach promised to decrease the morbidity related to traditional open spine surgery; the minimally invasive transpsoas approach started to become established as one of the preferred surgical approaches for lumbar interbody fusion.

Lateral lumbar interbody fusion has been used as a treatment option for multiple spinal disorders. It is a retroperitoneal approach that splits of the psoas muscle and avoids manipulation of the great vessels. ${ }^{1,21,32}$ However, the use of serial dilators and retractors to separate the psoas fibers has been implicated as a possible cause of injury to the lumbar plexus. ${ }^{1,5}$ In an analysis of 600 cases, Rodgers et al. reported $65 \%$ immediate improvement after the intervention. However, they also reported 37 complications $-60 \%$ medical and $40 \%$ surgical. While no wound infections, 
TABLE 3. Results of motor testing performed 60 minutes after the end of the positioning session*

\begin{tabular}{|c|c|c|c|c|c|c|c|}
\hline \multirow{2}{*}{$\begin{array}{c}\text { Subject } \\
\text { No. }\end{array}$} & \multirow{2}{*}{$\begin{array}{l}\text { Age } \\
\text { (yrs) }\end{array}$} & \multirow[b]{2}{*}{ Sex } & \multirow[b]{2}{*}{ Position } & \multicolumn{4}{|c|}{ PostP 10Max } \\
\hline & & & & RKE & LKE & RHF & LHF \\
\hline 1 & 29 & $\mathrm{~F}$ & RLD & $10 / 10$ & $10 / 10$ & $10 / 10$ & $10 / 10$ \\
\hline 2 & 33 & $M$ & RLJK & $10 / 10$ & $7 / 10$ & $10 / 10$ & $7 / 10$ \\
\hline 3 & 27 & M & RLJK & $10 / 10$ & $7 / 10$ & $10 / 10$ & $6 / 10$ \\
\hline 4 & 27 & $\mathrm{~F}$ & RLJK & $10 / 10$ & $8 / 10$ & $10 / 10$ & $6 / 10$ \\
\hline 5 & 28 & $\mathrm{~F}$ & RLD & $10 / 10$ & $10 / 10$ & $10 / 10$ & $10 / 10$ \\
\hline 6 & 28 & M & RLJK & $10 / 10$ & $10 / 10$ & $10 / 10$ & $7 / 10$ \\
\hline 7 & 26 & $M$ & RLJK & $10 / 10$ & $9 / 10$ & $10 / 10$ & $4 / 10$ \\
\hline 8 & 31 & M & RLD & $10 / 10$ & $10 / 10$ & $10 / 10$ & $10 / 10$ \\
\hline 9 & 30 & $\mathrm{~F}$ & RLD & $10 / 10$ & $10 / 10$ & $10 / 10$ & $10 / 10$ \\
\hline 10 & 25 & $M$ & RLD & $10 / 10$ & $10 / 10$ & $10 / 10$ & $10 / 10$ \\
\hline 11 & 29 & $\mathrm{~F}$ & RLJK & $10 / 10$ & $4 / 10$ & $10 / 10$ & $6 / 10$ \\
\hline 12 & 27 & $M$ & RLD & $10 / 10$ & $10 / 10$ & $10 / 10$ & $10 / 10$ \\
\hline 13 & 30 & $\mathrm{~F}$ & RLJK & $10 / 10$ & $7 / 10$ & $10 / 10$ & $8 / 10$ \\
\hline 14 & 27 & $M$ & RLJK & $10 / 10$ & $10 / 10$ & $10 / 10$ & $8 / 10$ \\
\hline 15 & 30 & M & RLD & $10 / 10$ & $10 / 10$ & $10 / 10$ & $10 / 10$ \\
\hline 16 & 26 & $M$ & RLD & $10 / 10$ & $10 / 10$ & $10 / 10$ & $10 / 10$ \\
\hline 17 & 27 & $M$ & RLD & $10 / 10$ & $10 / 10$ & $10 / 10$ & $10 / 10$ \\
\hline 18 & 28 & $F$ & RLJK & $10 / 10$ & $9 / 10$ & $10 / 10$ & $8 / 10$ \\
\hline 19 & 32 & $\mathrm{~F}$ & RLJK & $10 / 10$ & $9 / 10$ & $10 / 10$ & $9 / 10$ \\
\hline 20 & 34 & $\mathrm{~F}$ & RLD & $10 / 10$ & $10 / 10$ & $10 / 10$ & $10 / 10$ \\
\hline 21 & 21 & $\mathrm{~F}$ & RLJK & $10 / 10$ & $10 / 10$ & $10 / 10$ & $10 / 10$ \\
\hline 22 & 25 & $M$ & RLD & $10 / 10$ & $10 / 10$ & $10 / 10$ & $10 / 10$ \\
\hline 23 & 23 & $F$ & RLJK & $10 / 10$ & $10 / 10$ & $10 / 10$ & $10 / 10$ \\
\hline 24 & 30 & $\mathrm{~F}$ & RLD & $10 / 10$ & $10 / 10$ & $10 / 10$ & $10 / 10$ \\
\hline 25 & 25 & $\mathrm{~F}$ & RLD & $10 / 10$ & $10 / 10$ & $10 / 10$ & $10 / 10$ \\
\hline 26 & 29 & $M$ & RLJK & $10 / 10$ & $10 / 10$ & $10 / 10$ & $10 / 10$ \\
\hline 27 & 27 & $F$ & RLJK & $10 / 10$ & $9 / 10$ & $10 / 10$ & $9 / 10$ \\
\hline 28 & 31 & $\mathrm{~F}$ & RLD & $10 / 10$ & $10 / 10$ & $10 / 10$ & $10 / 10$ \\
\hline 29 & 31 & $M$ & RLJK & $10 / 10$ & $8 / 10$ & $10 / 10$ & $9 / 10$ \\
\hline 30 & 21 & $F$ & RLJK & $10 / 10$ & $8 / 10$ & $10 / 10$ & $8 / 10$ \\
\hline 31 & 25 & $\mathrm{~F}$ & RLJK & $10 / 10$ & $7 / 10$ & $10 / 10$ & $6 / 10$ \\
\hline 32 & 29 & $\mathrm{~F}$ & RLJK & $10 / 10$ & $9 / 10$ & $10 / 10$ & $10 / 10$ \\
\hline 33 & 27 & $\mathrm{~F}$ & RLJK & $10 / 10$ & $9 / 10$ & $10 / 10$ & $8 / 10$ \\
\hline 34 & 29 & $M$ & RLD & $10 / 10$ & $10 / 10$ & $10 / 10$ & $10 / 10$ \\
\hline 35 & 27 & $\mathrm{~F}$ & RLD & $10 / 10$ & $10 / 10$ & $10 / 10$ & $10 / 10$ \\
\hline 36 & 28 & $\mathrm{~F}$ & RLD & $10 / 10$ & $10 / 10$ & $10 / 10$ & $10 / 10$ \\
\hline 37 & 32 & $M$ & RLD & $10 / 10$ & $10 / 10$ & $10 / 10$ & $10 / 10$ \\
\hline 38 & 29 & $M$ & RLJK & $10 / 10$ & $10 / 10$ & $10 / 10$ & $10 / 10$ \\
\hline 39 & 28 & $M$ & RLD & $10 / 10$ & $10 / 10$ & $10 / 10$ & $10 / 10$ \\
\hline 40 & 28 & $M$ & RLD & $10 / 10$ & $10 / 10$ & $10 / 10$ & $10 / 10$ \\
\hline 41 & 27 & $F$ & RLD & $10 / 10$ & $10 / 10$ & $10 / 10$ & $10 / 10$ \\
\hline 42 & 29 & $M$ & RLJK & $10 / 10$ & $9 / 10$ & $10 / 10$ & $8 / 10$ \\
\hline 43 & 30 & $M$ & RLJK & $10 / 10$ & $9 / 10$ & $10 / 10$ & $10 / 10$ \\
\hline 44 & 31 & $M$ & RLD & $10 / 10$ & $10 / 10$ & $10 / 10$ & $10 / 10$ \\
\hline 45 & 27 & $M$ & RLD & $10 / 10$ & $10 / 10$ & $10 / 10$ & $10 / 10$ \\
\hline 46 & 26 & $M$ & RLD & $10 / 10$ & $10 / 10$ & $10 / 10$ & $10 / 10$ \\
\hline 47 & 29 & $\mathrm{~F}$ & RLD & $10 / 10$ & $10 / 10$ & $10 / 10$ & $10 / 10$ \\
\hline
\end{tabular}

(continued)
TABLE 3. Results of motor testing performed 60 minutes after the end of the positioning session* (continued)

\begin{tabular}{cccccccc}
\hline \multirow{2}{*}{$\begin{array}{c}\text { Subject } \\
\text { No. }\end{array}$} & Age & & & \multicolumn{4}{c}{ PostP 10Max } \\
\cline { 5 - 8 } & (yrs) & Sex & Position & RKE & LKE & RHF & LHF \\
\hline 48 & 29 & M & RLJK & $10 / 10$ & $10 / 10$ & $10 / 10$ & $10 / 10$ \\
\hline 49 & 35 & M & RLJK & $10 / 10$ & $10 / 10$ & $10 / 10$ & $10 / 10$ \\
\hline 50 & 31 & F & RLJK & $10 / 10$ & $10 / 10$ & $10 / 10$ & $9 / 10$ \\
\hline
\end{tabular}

* Shading indicates Group B (RLJK).

vascular injuries, or visceral injuries were described, 4 patients presented with quadriceps weakness, and hip flexion weakness was observed almost universally. These findings were observed despite neurological monitoring in all the cases. ${ }^{22}$ Ozgur et al. described similar results, reporting hip flexion weakness as the most frequent complication, which typically resolved within 6 weeks. For several years these symptoms have been attributed to the disruption of the psoas and a possible injury of the lumbar plexus, but the origin is not fully understood..$^{19}$

In the current study we tested the hypothesis that jackknife positioning alone could cause neural compromise and subsequent symptoms. Twenty-five of the 50 subjects recruited were positioned in the RLJK position, while the other half were in the lateral decubitus position. During the postpositioning evaluation, $100 \%$ of the subjects in the lateral jack-knife group (Group B) presented 20\%-80\% weakness in the left lower extremity when performing the hip flexion 10Max test, whereas none of the subjects in the lateral decubitus group (Group A) presented any degree of weakness. Similar findings were also observed during knee extension. In addition to the motor changes, alterations in sensory examination findings were also observed in Group B during the postpositioning pinprick $(92 \%$ in $\mathrm{L}-1,88 \%$ in L-2, and $44 \%$ in L-3) and light touch testing $(80 \%$ in L-1, $68 \%$ in L-2, and $52 \%$ in L-3). After the 60 -minute recovery time, we observed improvements in the ability to perform the 10Max test, and almost all of the subjects recovered from their initial sensory deficit. Ninety-eight percent of the individuals who participated in the study denied any symptoms during the follow-up call 7 days after the second visit (positioning day).

Extreme lateral interbody fusion is commonly used for a variety of conditions, with the average age of patients that undergo this procedure being between 60 and 70 years. ${ }^{1,25}$ For this study, we decided that the age of the participants should be younger (20-40 years old) and that the participants should not have any significant medical history, including but not limited to back problems and/ or neuropathies. Younger subjects with no comorbid conditions provide the best possible scenario to determine whether RLJK position alone contributes to cases of transient neurapraxia. Many patients who undergo lateral interbody fusion may have predisposing factors that make them more likely to develop neurapraxia. Nerve root irritation due to external compression, longer operative time, and advanced age are some of the aspects that could potentially contribute to the development of postoperative neurological deficits. We hypothesized that the presence of symptoms in young healthy volunteers could translate 
TABLE 4. Results of pinprick examination of left lower extremity in Group B

\begin{tabular}{|c|c|c|c|}
\hline \multirow{2}{*}{$\begin{array}{c}\text { Dermatome \& Time in } \\
\text { Minutes }^{*}\end{array}$} & \multicolumn{3}{|c|}{ Response } \\
\hline & Absent & Decreased & Normal \\
\hline \multicolumn{4}{|l|}{ L-1 } \\
\hline 0 & $12 \%$ & $80 \%$ & $8 \%$ \\
\hline 15 & $12 \%$ & $84 \%$ & $4 \%$ \\
\hline 30 & $4 \%$ & $76 \%$ & $20 \%$ \\
\hline 45 & 0 & $56 \%$ & $44 \%$ \\
\hline 60 & 0 & $36 \%$ & $64 \%$ \\
\hline \multicolumn{4}{|l|}{ L-2 } \\
\hline 0 & $12 \%$ & $76 \%$ & $12 \%$ \\
\hline 15 & $8 \%$ & $76 \%$ & $16 \%$ \\
\hline 30 & $4 \%$ & $68 \%$ & $28 \%$ \\
\hline 45 & $4 \%$ & $44 \%$ & $52 \%$ \\
\hline 60 & 0 & $20 \%$ & $80 \%$ \\
\hline \multicolumn{4}{|l|}{ L-3 } \\
\hline 0 & 0 & $44 \%$ & $56 \%$ \\
\hline 15 & 0 & $32 \%$ & $68 \%$ \\
\hline 30 & 0 & $24 \%$ & $76 \%$ \\
\hline 45 & 0 & $12 \%$ & $88 \%$ \\
\hline 60 & 0 & $8 \%$ & $92 \%$ \\
\hline \multicolumn{4}{|l|}{ L-4 } \\
\hline 0 & 0 & 0 & $100 \%$ \\
\hline 15 & 0 & 0 & $100 \%$ \\
\hline 30 & 0 & 0 & $100 \%$ \\
\hline 45 & 0 & 0 & $100 \%$ \\
\hline 60 & 0 & 0 & $100 \%$ \\
\hline
\end{tabular}

* Time elapsed since the end of the RLJK positioning session.

very well to the population undergoing lateral interbody fusion. Conditions such as diabetes, malnutrition, cancer, smoking, alcoholism, previous nerve injuries, and extreme weight are associated with higher incidence of positioning nerve injury.

Nerve injury due to patient positioning on the operating table was initially described in the 1800 s, most of them attributed to the patients' malposition. Despite the decrease in the incidence of these injuries $(0.02 \%-21 \%)$ through preventive measures, specific positions required for some procedures are associated with higher risk of neurapraxia. ${ }^{9}$ Peripheral nerve elongation/stretching and compression during surgery have proven to be responsible for postoperative neurological deficit. ${ }^{6}$ Nerve roots lack epineurium and perineurium and are therefore less resilient to stretch and compression..$^{12,18}$ Nerve roots have been found to be only $10 \%$ as strong as peripheral nerves and only $17 \%$ as stiff. "Small forces transmitted to roots via the nerve would cause substantial damage to roots and root attachments." 3 Animal in vivo testing on rat L-5 nerve roots has revealed strains of $16 \%, 10 \%$, and $9 \%$, at rates of $0.01 \mathrm{~mm} / \mathrm{sec}, 1 \mathrm{~mm} / \mathrm{sec}$, and $15 \mathrm{~mm} / \mathrm{sec}$, respectively, led to a $50 \%$ probability of complete conduction block. Spinal nerve root tissue exhibits an increase in functional deficit with increasing strain but at lower rates of distraction than
TABLE 5. Results of light touch examination in Group B

\begin{tabular}{|c|c|c|c|}
\hline \multirow{2}{*}{$\begin{array}{c}\text { Dermatome \& Time in } \\
\text { Minutes }^{*}\end{array}$} & \multicolumn{3}{|c|}{ Response } \\
\hline & Absent & Decreased & Normal \\
\hline \multicolumn{4}{|l|}{$L-1$} \\
\hline 0 & $12 \%$ & $68 \%$ & $20 \%$ \\
\hline 15 & 0 & $80 \%$ & $20 \%$ \\
\hline 30 & 0 & $67 \%$ & $33 \%$ \\
\hline 45 & 0 & $40 \%$ & $60 \%$ \\
\hline 60 & 0 & $36 \%$ & $64 \%$ \\
\hline \multicolumn{4}{|l|}{$\mathrm{L}-2$} \\
\hline 0 & $12 \%$ & $56 \%$ & $32 \%$ \\
\hline 15 & $4 \%$ & $60 \%$ & $36 \%$ \\
\hline 30 & $4 \%$ & $48 \%$ & $48 \%$ \\
\hline 45 & $4 \%$ & $28 \%$ & $68 \%$ \\
\hline 60 & 0 & $12 \%$ & $88 \%$ \\
\hline \multicolumn{4}{|l|}{ L-3 } \\
\hline 0 & 0 & $52 \%$ & $48 \%$ \\
\hline 15 & 0 & $44 \%$ & $56 \%$ \\
\hline 30 & 0 & $28 \%$ & $72 \%$ \\
\hline 45 & 0 & $12 \%$ & $88 \%$ \\
\hline 60 & 0 & $8 \%$ & $92 \%$ \\
\hline \multicolumn{4}{|l|}{ L-4 } \\
\hline 0 & 0 & $8 \%$ & $92 \%$ \\
\hline 15 & 0 & $4 \%$ & $96 \%$ \\
\hline 30 & 0 & 0 & $100 \%$ \\
\hline 45 & 0 & 0 & $100 \%$ \\
\hline 60 & 0 & 0 & $100 \%$ \\
\hline
\end{tabular}

* Time elapsed since the end of the RLJK positioning session.

seen in peripheral nerve studies. ${ }^{29}$ Neural stretch injuries have been found to cause a wide variety of pathophysiological changes depending on the amount of stretch. Animal models have shown impairment of peripheral nerve blood flow induced with an $8 \%$ increase of in vivo length. Complete intraneural ischemia is induced at $15 \%$ increase in length. ${ }^{12,18}$ Peripheral nerve mechanical failure occurs at $27 \%$ increase in length. ${ }^{24}$ Decrease in blood flow causing patching ischemia and/or tear of the intraneural connecting tissue causing hemorrhage and/or necrosis are considered the pathophysiology behind the symptoms. Continuous high levels of compression or elongation could lead to an increase of the intraneural venous pressure, which produces endoneural edema. ${ }^{30}$ In more severe cases, myelin displacement, Schwann cell damage, and Wallerian degeneration are observed, mostly resulting in a poor prognosis. ${ }^{36} \mathrm{Up}$ to $70 \%$ loss of nerve action potential (NAP) amplitudes can be detected during 6\% strain for one hour. These functional changes have shown to be reversible. However, at $12 \%$ strain for 1 hour, complete conduction block was noted with minimal recovery. ${ }^{33}$ Irreversible loss of nerve function has been reported with $16 \%$ strain to a peripheral nerve. ${ }^{6}$

In addition to the operating time, which has been related to higher incidence of postoperative nerve injury, the type 
of surgery has been found to be another determining factor. ${ }^{9}$ Welch et al. found orthopedic surgeries to carry the highest risk of operative nerve injury (21\%), followed by general surgery (18\%), neurological surgery $(8.9 \%)$, and cardiac surgery (7.1\%). Other studies found a higher correlation of upper-extremity neurapraxia after cardiac surgery, but the highest risk is still related to orthopedic surgeries. ${ }^{26}$

In the case of lateral interbody fusion procedures, the L4-5 intervertebral disc is the most challenging when using a transpsoas approach. This is due to the iliac crest and the course and size of the obturator and femoral nerves. ${ }^{5,16}$ A lateral jack-knife position is used to drop the ipsilateral iliac crest and expose the L4-5 level under fluoroscopic guidance. ${ }^{21}$ Intraforaminal ligaments create a point of nerve root sheath fixation which may contribute to neural traction forces during positioning and then again during retractor placement and expansion. ${ }^{11}$ Ipsilateral hip flexion has been recommended to decrease the amount of traction on the femoral nerve during the procedure. ${ }^{32}$ During the present study, all subjects in Group B (RLJK) exhibited a decrease in the postpositioning 10Max in the nondependent lower limb (knee extension and hip flexion) in addition to alterations to sensory testing results in $98 \%$ of the subjects. These findings were statistically significant when compared with the results obtained in Group A (RLD), in which the subjects did not exhibit significant changes. There were no differences found between males and females with respect to results of testing. A significant decrease in 10Max results for knee extension was observed in 100\% of Group B (RLJK) subjects during postpositioning testing. The findings from this study, appear to explain the high percentage of sensory disturbances reported in prospective studies after transpsoas approach but present a significantly higher incidence of motor deficits. ${ }^{1,19}$ The reason for this discrepancy is likely due to the low sensitivity of manual muscle testing compared with the 10Max testing that we used. ${ }^{4}$ Beasley reported manual muscle testing of knee extension strength to be considered normal when in fact it was $50 \%$ decreased. ${ }^{2}$ Three-nerve cross-innervation as well as the combination of 4 muscles working in symphony for knee extension enables the quadriceps group be resilient and likely contributes to the decreased sensitivity of manual muscle testing.

The $25^{\circ}$ angulation of the operating table for the lateral jack-knife position translates to lateral flexion of the lumbar spine. Fazey et al. studied the biomechanical changes of the lumbar spine during lateral flexion. Intersegmental angles were calculated using the lines extending from the anterior and posterior corners of L-1 to L-5 and the midpoint where they intersected. The results of this study revealed statistically significant differences $(p<0.005)$ within the angles formed at the different lumbar vertebral levels, with the greatest lexion being at the L3-4 $\left(6.7^{\circ}\right)$ and L4 $-5\left(6.7^{\circ}\right)$ levels. These findings make L-4 the vertex of the angle formed in the lumbar spine during lateral flexion. ${ }^{8}$ These biomechanical results can be correlated to the findings of our study. Lumbar nerve roots originating above the L-4 vertebral body (L-1 to L-3) are exposed to higher levels of stretch in a laterally flexed position. The L-5 nerve, which exits below the L-4 vertebra, is subjected to less traction in this position. This is the most likely explanation as to why postpositioning neural symptoms were seen in the L-1, L-2, L-3 dermatomes and no changes were observed at L-5.

During this study, young healthy conscious subjects began to experience sensory changes after 30 minutes in the jack-knife position at $25^{\circ}$ and experienced motor and sensory deficits after 60 minutes. All strength testing was performed by the same blinded physical therapist. Sensory testing was performed by the same nonblinded physician. When considering these finding as a baseline concept, this would likely be compounded in older subjects with comorbid issues and less resilient neural systems. When considering these findings with respect to clinical practice, it is important to remember that the effects are likely to be compounded in older individuals with comorbid conditions and less resilient neural systems. They would also be compounded in a patient under anesthesia unable to adjust position in response to discomfort and during hypotensive anesthesia, causing less oxygenation to the neural structures that are already at a disadvantage. The results of this study suggest that avoiding the lateral jack-knife position during transpsoas lumbar surgery could decrease postoperative neurological symptoms. In cases in which the patient is required to remain in the jack-knife position in order to provide access to L4-5, time is of the essence to avoid prolonged neural stretch. Once again, in this study, conscious subjects began to experience sensory deficits at 30 minutes. If the surgical case is lengthy, one might consider releasing the jack-knife temporarily to allow a recovery time for the neural structures. For a multilevel case, one might consider using the jack-knife position to access the L4-5 disc, then releasing the jack-knife for subsequent levels, allowing for release of traction to the lumbar roots.

\section{Conclusions}

One hundred percent of healthy volunteers who were maintained in a lateral jack-knife position for 1 hour (Group B) exhibited variable degrees of neurapraxia in the nondependent lower limb during testing after the 1-hour session. All had motor deficits and $98 \%$ had sensory deficits. These results starkly contrast with those obtained from the volunteers who were placed in the lateral decubitus position (Group A), none of whom had any significant neurological signs or symptoms. These findings confirm a direct relationship between the lateral jack-knife position and transient neurapraxia in the lumbar plexus. This study supports the hypothesis that jack-knife positioning alone can contribute to postoperative neurapraxia in the nondependent ("upside") lower limb.

\section{References}

1. Arnold PM, Anderson KK, McGuire RA Jr: The lateral transpsoas approach to the lumbar and thoracic spine: A review. Surg Neurol Int 3 (Suppl 3):S198-S215, 2012

2. Beasley WC: Influence of method on estimates of normal knee extensor force among normal and postpolio children. Phys Ther Rev 36:21-41, 1956

3. Beel JA, Stodieck LS, Luttges MW: Structural properties of spinal nerve roots: biomechanics. Exp Neurol 91:30-40, 1986

4. Bohannon RW: Measuring knee extensor muscle strength. Am J Phys Med Rehabil 80:13-18, 2001 
5. Davis TT, Bae HW, Mok JM, Rasouli A, Delamarter RB: Lumbar plexus anatomy within the psoas muscle: implications for the transpsoas lateral approach to the L4-L5 disc. J Bone Joint Surg Am 93:1482-1487, 2011

6. Driscoll PJ, Glasby MA, Lawson GM: An in vivo study of peripheral nerves in continuity: biomechanical and physiological responses to elongation. J Orthop Res 20:370-375, 2002

7. Faust RJ, Cucchiara RF, Bechtle PS: Patient positioning, in Miller R (ed): Miller's Anesthesia, ed 6. Philadelphia: Elsevier, 2005, pp 1151-1167

8. Fazey PJ, Takasaki H, Singer KP: Nucleus pulposus deformation in response to lumbar spine lateral flexion: an in vivo MRI investigation. Eur Spine J 19:1115-1120, 2010

9. Ferullo SB: Preventing perioperative peripheral nerve injuries. AORN J 97:110-124, 2013

10. Fish DE, Krabak BJ, Johnson-Greene D, DeLateur BJ: Optimal resistance training: comparison of DeLorme with Oxford techniques. Am J Phys Med Rehabil 82:903-909, 2003

11. Grimes PF, Massie JB, Garfin SR: Anatomic and biomechanical analysis of the lower lumbar foraminal ligaments. Spine (Phila Pa 1976) 25:2009-2014, 2000

12. Haller FR, Low FN: The fine structure of the peripheral nerve root sheath in the subarachnoid space in the rat and other laboratory animals. Am J Anat 131:1-19, 1971

13. Houten JK, Alexandre LC, Nasser R, Wollowick AL: Nerve injury during the transpsoas approach for lumbar fusion. $\mathbf{J}$ Neurosurg Spine 15:280-284, 2011

14. Isaacs RE, Hyde J, Goodrich JA, Rodgers WB, Phillips FMA: A prospective, nonrandomized, multicenter evaluation of extreme lateral interbody fusion for the treatment of adult degenerative scoliosis: perioperative outcomes and complications. Spine (Phila Pa 1976) 35 (26 Suppl):S322-S330, 2010

15. Kamel IR, Drum ET, Koch SA, Whitten JA, Gaughan JP, Barnette RE, et al: The use of somatosensory evoked potentials to determine the relationship between patient positioning and impending upper extremity nerve injury during spine surgery: a retrospective analysis. Anesth Analg 102:15381542,2006

16. Kepler CK, Bogner EA, Herzog RJ, Huang RC: Anatomy of the psoas muscle and lumbar plexus with respect to the surgical approach for lateral transpsoas interbody fusion. Eur Spine J 20:550-556, 2011

17. Lundborg G, Rydevik B: Effects of stretching the tibial nerve of the rabbit. A preliminary study of the intraneural circulation and the barrier function of the perineurium. J Bone Joint Surg Br 55:390-401, 1973

18. McCabe JS, Low FN: The subarachnoid angle: an area of transition in peripheral nerve. Anat Rec 164:15-33, 1969

19. Moller DJ, Slimack NP, Acosta FL Jr, Koski TR, Fessler RG, Liu JC: Minimally invasive lateral lumbar interbody fusion and transpsoas approach-related morbidity. Neurosurg Focus 31(4):E4, 2011

20. Ozgur BM, Agarwal V, Nail E, Pimenta L: Two-year clinical and radiographic success of minimally invasive lateral transpsoas approach for the treatment of degenerative lumbar conditions. SAS J 4:41-46, 2010

21. Ozgur BM, Aryan HE, Pimenta L, Taylor WR: Extreme Lateral Interbody Fusion (XLIF): a novel surgical technique for anterior lumbar interbody fusion. Spine J 6:435-443, 2006

22. Rodgers WB, Cox CS, Gerber EJ: Early complications of extreme lateral interbody fusion in the obese. J Spinal Disord Tech 23:393-397, 2010

23. Rodgers WB, Gerber EJ, Patterson J: Intraoperative and early postoperative complications in extreme lateral interbody fusion: an analysis of 600 cases. Spine (Phila Pa 1976) 36:2632,2011

24. Rydevik BL, Kwan MK, Myers RR, Brown RA, Triggs KJ, Woo SL, et al: An in vitro mechanical and histological study of acute stretching on rabbit tibial nerve. J Orthop Res 8:694-701, 1990

25. Schonauer C, Bocchetti A, Barbagallo G, Albanese V, Moraci A: Positioning on surgical table. Eur Spine J 13 (Suppl 1):S50-S55, 2004

26. Seyfer AE, Grammer NY, Bogumill GP, Provost JM, Chandry U: Upper extremity neuropathies after cardiac surgery. J Hand Surg Am 10:16-19, 1985

27. Sharma AK, Kepler CK, Girardi FP, Cammisa FP, Huang RC, Sama AA: Lateral lumbar interbody fusion: clinical and radiographic outcomes at 1 year: a preliminary report. J Spinal Disord Tech 24:242-250, 2011

28. Shen FH, Samartzis D, Khanna AJ, Anderson DG: Minimally invasive techniques for lumbar interbody fusions. Orthop Clin North Am 38:373-386, 2007 (Abstract \#6)

29. Singh A, Kallakuri S, Chen C, Cavanaugh JM: Structural and functional changes in nerve roots due to tension at various strains and strain rates: an in-vivo study. J Neurotrauma 26:627-640, 2009

30. Stewart JD: Focal Peripheral Neuropathies, ed 3. Philadelphia: Lippincott Williams \& Wilkins, 2000

31. Tohmeh AG, Rodgers WB, Peterson MD: Dynamically evoked, discrete-threshold electromyography in the extreme lateral interbody fusion approach. J Neurosurg Spine 14:31-37, 2011

32. Tormenti MJ, Maserati MB, Bonfield CM, Okonkwo DO, Kanter AS: Complications and radiographic correction in adult scoliosis following combined transpsoas extreme lateral interbody fusion and posterior pedicle screw instrumentation. Neurosurg Focus 28(3):E7, 2010

33. Wall EJ, Massie JB, Kwan MK, Rydevik BL, Myers RR, Garfin SR: Experimental stretch neuropathy. Changes in nerve conduction under tension. J Bone Joint Surg Br 74:126-129, 1992

34. Walsworth M, Schneider R, Schultz I, Da hl C, Allison S, Underwood F, et al: Prediction of 10 repetition maximum for short-arc quadriceps exercise from hand-held dynamometer and anthropometric measurement. J Orthop Sports Phys Ther 28:97-104, 1998

35. Welch MB, Brummett CM, Welch TD, Tremper KK, Shanks AM, Guglani P, et al: Perioperative peripheral nerve injuries: a retrospective study of 380,680 cases during a 10 -year period at a single institution. Anesthesiology 111:490-497, 2009

36. Winfree CJ, Kline DG: Intraoperative positioning nerve injuries. Surg Neurol 63:5-18, 2005

37. Yang SH, Wu CC, Chen PQ: Postoperative meralgia paresthetica after posterior spine surgery: incidence, risk factors, and clinical outcomes. Spine (Phila Pa 1976) 30:E547E550, 2005

\section{Disclosure}

Support for this clinical study was received from Medtronic Sofamor Danek USA.

\section{Author Contributions}

Conception and design: Fung, Molinares, Davis, Mok. Acquisition of data: Molinares, Mok. Analysis and interpretation of data: Fung, Molinares. Drafting the article: Fung, Molinares. Critically revising the article: Fung, Molinares, Davis, Liu. Reviewed submitted version of manuscript: all authors. Approved the final version of the manuscript on behalf of all authors: Fung. Statistical analysis: Molinares, Davis. Administrative/technical/material support: Davis, Clark, Daily. Study supervision: Fung, Davis.

\section{Correspondence}

Daniel A. Fung, Orthopedic Pain Specialists, 2811 Wilshire Blvd., Ste.850, Santa Monica, CA 90403.email: dfungmd@ gmail.com. 